\title{
An Optimization-Based Sum-of-Squares Approach to Vizing's Conjecture
}

\author{
Elisabeth Gaar \\ Angelika Wiegele \\ elisabeth.gaar@aau.at \\ angelika.wiegele@aau.at \\ Alpen-Adria-Universität \\ Klagenfurt, Austria
}

\author{
Daniel Krenn \\ Uppsala Universitet \\ Uppsala, Sweden \\ math@danielkrenn.at
}

\author{
Susan Margulies \\ United States Naval Academy \\ Annapolis, MD, USA \\ margulie@usna.edu
}

\begin{abstract}
Vizing's conjecture (open since 1968) relates the sizes of dominating sets in two graphs to the size of a dominating set in their Cartesian product graph. In this paper, we formulate Vizing's conjecture itself as a Positivstellensatz existence question. In particular, we encode the conjecture as an ideal/polynomial pair such that the polynomial is nonnegative if and only if the conjecture is true. We demonstrate how to use semidefinite optimization techniques to computationally obtain numeric sum-of-squares certificates, and then show how to transform these numeric certificates into symbolic certificates approving nonnegativity of our polynomial.

After outlining the theoretical structure of this computer-based proof of Vizing's conjecture, we present computational and theoretical results. In particular, we present exact low-degree sparse sum-of-squares certificates for particular families of graphs.
\end{abstract}

\section{KEYWORDS}

Vizing's conjecture; algebraic model; Gröbner basis; sum-of-squares problems; semidefinite programming

\section{ACM Reference Format:}

Elisabeth Gaar, Daniel Krenn, Susan Margulies, and Angelika Wiegele. 2019. An Optimization-Based Sum-of-Squares Approach to Vizing's Conjecture. In International Symposium on Symbolic and Algebraic Computation (ISSAC '19), July 15-18, 2019, Beijing, China. ACM, New York, NY, USA, 8 pages. https://doi.org/10.1145/3326229.3326239

\section{INTRODUCTION}

Sum-of-squares and its relationship to semidefinite programming is a cutting-edge tool at the forefront of polynomial optimization [5]. Activity in this area has exploded over the past two decades to span areas as diverse as real and convex algebraic geometry [17], control theory [15], proof complexity [12], theoretical computer science [2] and even quantum computation [3]. Systems of polynomial equations and other non-linear models are similarly widely known for their compact and elegant representations of combinatorial problems. Prior work on polynomial encodings includes colorings [1,14], stable sets [18, 19], matchings [9], and flows [22]. In this project, we combine the modeling strength of systems of

This paper is published under a Creative Commons Attribution 4.0 International License (http://creativecommons.org/licenses/by/4.0/)

(c) BY

ISSAC '19, July 15-18, 2019, Beijing, China

(C) 2019 Copyright held by the authors.

ACM ISBN 978-1-4503-6084-5/19/07.

https://doi.org/10.1145/3326229.3326239 polynomial equations with the computational power of semidefinite programming and devise an optimization-based framework for a computational proof of an old, open problem in graph theory, namely Vizing's conjecture.

Vizing's conjecture was first proposed in 1968, and relates the sizes of minimum dominating sets in graphs $G$ and $H$ to the size of a minimum dominating set in the Cartesian product graph $G \square H$; a precise formulation follows as Conjecture 2.1. Prior algebraic work on this conjecture [20] expressed the problem as the union of a certain set of varieties and thus the intersection of a certain set of ideals. However, algebraic computational results have remained largely untouched. In this project, we present an algebraic model of Vizing's conjecture that equates the validity of the conjecture to the existence of a Positivstellensatz, or a sum-of-squares certificate of nonnegativity modulo a carefully constructed ideal.

By exploiting the relationship between the Positivstellensatz and semidefinite programming, we are able to produce sum-of-squares certificates for certain classes of graphs where Vizing's conjecture holds. Thus, not only are we demonstrating an optimization-based approach towards a computational proof of Vizing's conjecture, but we are presenting actual minimum degree nonnegativity certificates that are algebraic proofs of instances of this combinatorial problem. Although the underlying graphs do not further what is known about Vizing's conjecture at this time (indeed the combinatorics of the underlying graphs is fairly trivial), the construction of these "combinatorial" Positivstellensätze is an elegant combination of computation, guesswork and computer algebra that is successfully executed for the first time here.

Our paper is structured as follows. In Section 2, we present the necessary background and definitions from graph theory and commutative algebra. In Section 3, we begin the heart of the paper: we describe the ideal/polynomial pair that models Vizing's conjecture as a sum-of-squares problem. In Section 4 we describe our precise process for finding the sum-of-squares certificates, and in Section 5 we present our computational results and the Positivstellensätze, i.e., the theorems that arise. Finally, in Section 6, we summarize our project and present comments about future work.

\section{BACKGROUNDS AND DEFINITIONS}

\subsection{Definitions from Graph Theory}

Given a graph $G$ with vertex set $V(G)$, a set $D \subseteq V(G)$ is a dominating set in $G$ if for each $v \in V(G) \backslash D$, there is a $u \in D$ such that $v$ is 
adjacent to $u$ in $G$. The domination number of $G$, denoted by $\gamma(G)$, is the size of a minimum ${ }^{1}$ dominating set in $G$.

Given graphs $G$ and $H$ with edge sets $E(G)$ and $E(H)$, the Cartesian product graph $G \square H$ has vertex set $V(G) \times V(H)$ and edge set

$$
\begin{aligned}
E(G \square H)=\left\{\left(g h, g^{\prime} h^{\prime}\right):\right. & g=g^{\prime} \text { and }\left(h, h^{\prime}\right) \in E(H), \text { or } \\
h & \left.=h^{\prime} \text { and }\left(g, g^{\prime}\right) \in E(G)\right\},
\end{aligned}
$$

where $g, g^{\prime} \in V(G)$ and $h, h^{\prime} \in V(H)$.

In 1968 , V. Vizing conjectured the following beautiful relationship between domination numbers and Cartesian product graphs:

Conjecture 2.1 (Vizing [25], 1968). Graphs $G$ and H satisfy

$$
\gamma(G) \gamma(H) \leq \gamma(G \square H) .
$$

Example 2.2. In this example, we demonstrate the Cartesian product graph of two $C_{4}$ cycle graphs:

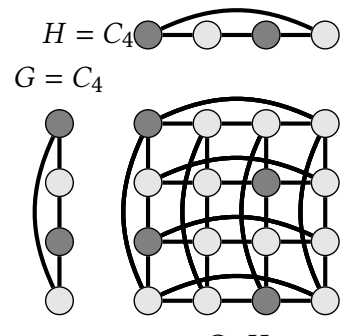

$$
G \square H
$$

In these graphs, $\bigcirc$ represents a vertex in a dominating set, and Vizing's conjecture holds with equality: $\gamma(G) \gamma(H)=2 \cdot 2=4=$ $\gamma(G \square H)$. However, observe that some copies of $G$ in $G \square H$ do not contain any vertices of the dominating set, i.e., they are dominated entirely by vertices in other "layers" of the graph. This example highlights the difficulty of Vizing's conjecture.

\subsection{Historical Notes}

Vizing's conjecture is an active area of research spanning over fifty years. Early results have focused on proving the conjecture for certain classes of graphs. For example, in 1979, Barcalkin and German [4] proved that Vizing's conjecture holds for graphs satisfying a certain "partitioning condition" on the vertex set. The idea of a "partitioning condition" inspired work for the next several decades, as Vizing's conjecture was shown to hold on paths, trees, cycles, chordal graphs, graphs satisfying certain coloring properties, and graphs with $\gamma(G) \leq 2$. These results are clearly outlined in the 1998 survey paper by Hartnell and Rall [13]. In 2000, Clark and Suen [7] showed that $\gamma(G) \gamma(H) \leq 2 \gamma(G \square H)$, and in 2004, Sun [23] showed that Vizing's conjecture holds on graphs with $\gamma(G) \leq 3$. Finally, the 2009 survey paper [6] summarizes the work from 1968 to 2008, contains new results, new proofs of existing results, and comments about minimal counter-examples.

\subsection{Definitions around Polynomial Ideals}

Our goal is to model Vizing's conjecture as a semidefinite programming problem. In particular, we will create an ideal/polynomial pair such that the polynomial is nonnegative over a given variety if and only if Vizing's conjecture is true.

\footnotetext{
${ }^{1}$ Any proper subset of a minimum dominating set is not dominating.
}

In this subsection, we present a brief introduction to polynomial ideals, and the relationship between nonnegativity and sum-ofsquares. This material is necessary for understanding our polynomial ideal model of Vizing's conjecture. For a more thorough introduction to this material see [8] and [5].

Throughout this section, let $I$ be an ideal in a polynomial $\operatorname{ring} P=$ $\mathbb{K}\left[x_{1}, \ldots, x_{n}\right]$ with a field $\mathbb{K} \subseteq \mathbb{R}$. The variety of the ideal $I$ is defined as the set

$$
\mathcal{V}(I)=\left\{z \in \overline{\mathbb{K}}^{n}: f(z)=0 \text { for all } f \in I\right\}
$$

with $\overline{\mathbb{K}}$ being the algebraic closure of $\mathbb{K}$. The variety $\mathcal{V}(I)$ is called real if $\mathcal{V}(I) \subseteq \mathbb{R}^{n}$.

We say that the ideal $I$ is radical if whenever $f^{m} \in I$ for some polynomial $f \in P$ and integer $m \geq 1$, then $f \in I$. The radical of $I$, denoted $\sqrt{I}$, is the set

$$
\sqrt{I}=\left\{f \in P: f^{m} \in I \text { for some integer } m \geq 1\right\} .
$$

It is easy to see that an ideal $I$ is radical if and only if $I=\sqrt{I}$.

Lemma 2.3. ([16, Section 3.7.B, pg. 246]) Given an ideal I with finite variety $\mathcal{V}(I)$, if I contains a univariate square-free polynomial in each variable, then I is radical.

In this case, square-free implies that when a polynomial is decomposed into its unique factorization, there are no repeated factors.

In particular, Lemma 2.3 implies that ideals containing $x_{i}^{2}-x_{i}=$ $x_{i}\left(x_{i}-1\right)$ in each variable (i.e., the boolean ideals) are radical.

Definition 2.4. Let $\ell$ be a nonnegative integer. A polynomial $f \in P$ is called $\ell$-sum-of-squares modulo $I$ (or $\ell$-sos $\bmod I$ ), if there exist polynomials $s_{1}, \ldots, s_{d} \in P$ with degrees deg $s_{i} \leq \ell$ for all $i \in\{1, \ldots, d\}$ and $f \equiv \sum_{i=1}^{d} s_{i}^{2} \bmod I$.

Algebraic identities like $f=\sum_{i=1}^{d} s_{i}^{2}+g, g \in I$, are often referred to as Positivstellensatz certificates of nonnegativity, and these identities can be found via semidefinite programming, which is at the heart of this project. It is well-known that not all nonnegative polynomials can be expressed as a sum-of-squares. However, in the particular case when the ideal is radical and the variety is finite, we can state the following.

LEMMA 2.5. Given a radical ideal I with a finite real variety and a polynomial $f$ with $f(\mathcal{V}(I)) \subseteq \mathbb{R}$. Then $f$ is nonnegative on the variety, i.e, $\forall z \in \mathcal{V}(I): f(z) \geq 0$, if and only if there exists a nonnegative integer $\ell$ such that $f$ is $\ell$-sos modulo $I$.

Proof. Let $f$ be a polynomial that can be expressed as a sumof-squares modulo $I$. Since all polynomials in the ideal $I$ vanish on the variety by definition and since $\sum_{i=1}^{d} s_{i}^{2}$ is clearly positive, $f$ is nonnegative on $\mathcal{V}(I)$. To prove the other direction, we recall a well-known argument included here for completeness. Suppose we have a polynomial $f(z) \geq 0$ for all $z \in \mathcal{V}(I)$. Suppose further that $\left\{z_{1}, \ldots, z_{t}\right\}$ are all points in $\mathcal{V}(I)$ (recall that the variety is finite). We now construct $t$ interpolation polynomials (see [10]) such that

$$
f_{i}(z)= \begin{cases}1 & z=z_{i}, \\ 0 & z \neq z_{i}\end{cases}
$$

for all $z \in \mathcal{V}(I)$. Observe that the square of an interpolating polynomial is again an interpolating polynomial. Since the ideal is radical, 
this means that $I=I(\mathcal{V}(I))$ where $I(\mathcal{V}(I))$ is the ideal vanishing on $\mathcal{V}(I)$. In this case, we see that the difference polynomial

$$
\left(f(z)-\sum_{i=1}^{t} f_{i}^{2}(z) f\left(z_{i}\right)\right) \in I
$$

since this difference polynomial vanishes on every point in the variety. Therefore, if we let $s_{i}=\sqrt{f\left(z_{i}\right)} f_{i}(z)$, we then see that

$$
f \equiv \sum_{i=1}^{d} s_{i}^{2} \bmod I .
$$

We observe that the $\ell$ in this case is quite large, since it is the degree of the interpolating polynomial $f_{i}$, which depends on the number of points in the variety. However, we will rely on the fact that the sum-of-squares representation is not unique, and there may exist Positivstellensatz certificates of much lower degree, within reach of computation. As we will see in Section 5, this does indeed turn out to be the case.

\section{VIZING'S CONJECTURE AS A SUM-OF-SQUARES PROBLEM}

In this section, we describe Vizing's conjecture as a sum-of-squares problem. Towards that end, we will first define ideals associated with graphs $G, H$ and $G \square H$, and then finally describe an ideal/ polynomial pair where the polynomial is nonnegative on the variety of the ideal if and only if Vizing's conjecture is true. We begin by creating an ideal where the variety of solutions corresponds to graphs with a given number of vertices and size of a minimum dominating set.

The notation underlying all of the definitions in this section is as follows. Let $n_{\mathcal{G}}$ and $k_{\mathcal{G}} \leq n_{\mathcal{G}}$ be fixed positive integers, and let $\mathcal{G}$ be the class of graphs on $n_{\mathcal{G}}$ vertices with a minimum dominating set $D_{\mathcal{G}}$ (fixed) of size $k_{\mathcal{G}}$. We then turn the various edges "on" or "off" (by controlling a boolean variable $e_{g g^{\prime}}$ ) such that each point in the variety corresponds to a specific graph $G \in \mathcal{G}$.

Definition 3.1. Set $e_{\mathcal{G}}=\left\{e_{g g^{\prime}}:\left\{g, g^{\prime}\right\} \subseteq V(\mathcal{G})\right\}$. The ideal $I_{\mathcal{G}} \subseteq P_{\mathcal{G}}=\mathbb{K}\left[e_{\mathcal{G}}\right]$ is defined by the system of polynomial equations

$$
\begin{array}{rlrl}
e_{g g^{\prime}}^{2}-e_{g g^{\prime}} & =0 & & \text { for }\left\{g, g^{\prime}\right\} \subseteq V(\mathcal{G}) \\
\prod_{g^{\prime} \in D_{\mathcal{G}}\left(1-e_{g g^{\prime}}\right)}=0 & & \text { for } g \in V(\mathcal{G}) \backslash D_{\mathcal{G}} \\
\prod_{g^{\prime} \in V(\mathcal{G}) \backslash S}\left(\sum_{g \in S} e_{g g^{\prime}}\right) & =0 & & \text { for } S \subseteq V(\mathcal{G}) \text { where }|S|=k_{\mathcal{G}}-1
\end{array}
$$

THEOREM 3.2. The points in the variety $\mathcal{V}\left(I_{\mathcal{G}}\right)$ are in bijection to the graphs in $\mathcal{G}$.

Proof. Consider any point $z \in \mathcal{V}\left(I_{\mathcal{G}}\right)$. Since Eqns. (1a) turn the edges "on" $\left(e_{g g^{\prime}}=1\right)$ or "off" $\left(e_{g g^{\prime}}=0\right)$, the point $z$ defines a graph $G$ in $n_{\mathcal{G}}$ vertices. Eqns. (1b) iterate over all the vertices inside the set $D_{\mathcal{G}}$, and ensure that for each vertex outside the set at least one edge from a vertex inside the set to this vertex is "on". Therefore, $D_{\mathcal{G}}$ is a dominating set. Finally, Eqns. (1c) iterate over all sets $S$ of size $k_{\mathcal{G}}-1$ and ensure that at least one vertex outside $S$ is not incident on any vertex inside $S$ for any $S$. Therefore, no $S$ of size
$k_{\mathcal{G}}-1$ is a dominating set. Thus, every point $z \in \mathcal{V}\left(I_{\mathcal{G}}\right)$ corresponds to a graph $G$ on $n_{\mathcal{G}}$ vertices with a minimum dominating set of size $k_{\mathcal{G}}$.

Similarly, for fixed positive integers $n_{\mathcal{H}}$ and $k_{\mathcal{H}} \leq n_{\mathcal{H}}$, let $\mathcal{H}$ be the class of graphs on $n_{\mathcal{H}}$ vertices and a minimum dominating set of size $k_{\mathcal{H}}$. Again, we fix the dominating set to some $D_{\mathcal{H}}$ to reduce isomorphisms within the variety. Furthermore let the ideal $I_{\mathcal{H}}$ be defined on the polynomial ring $P_{\mathcal{H}}=\mathbb{K}\left[e_{\mathcal{H}}\right]$ with $e_{\mathcal{H}}=\left\{e_{h h^{\prime}}\right.$ : $\left.\left\{h, h^{\prime}\right\} \subseteq V(\mathcal{H})\right\}$ such that the solutions in the variety $\mathcal{V}\left(I_{\mathcal{H}}\right)$ are in bijection to the graphs in $\mathcal{H}$.

Next, we define the graph class $\mathcal{G} \square \mathcal{H}$ and the ideal $I_{\mathcal{G} \square \mathcal{H}}$. For the above classes $\mathcal{G}$ and $\mathcal{H}$, the graph class $\mathcal{G} \square \mathcal{H}$ is the set of product graphs $G \square H$ for $G \in \mathcal{G}$ and $H \in \mathcal{H}$. The new variables needed for the ideal are the variables corresponding to the vertices in the product graph. Let $x_{\mathcal{G} \square \mathcal{H}}=\left\{x_{g h}: g \in V(\mathcal{G}), h \in V(\mathcal{H})\right\}$ and set $P_{\mathcal{G} \square \mathcal{H}}=\mathbb{K}\left[e_{\mathcal{G}} \cup e_{\mathcal{H}} \cup x_{\mathcal{G} \square \mathcal{H}}\right]$.

Definition 3.3. The ideal $I_{\mathcal{G} \square \mathcal{H}} \subseteq P_{\mathcal{G} \square \mathcal{H}}$ is defined by the system of polynomial equations

$$
\left(1-x_{g h}\right) \times
$$

for $g \in V(\mathcal{G})$ and $h \in V(\mathcal{H})$.

Observe that we have no restrictions on the edge variables in this definition. It is only used as a stepping stone to the final and most important ideal in our polynomial model.

Definition 3.4. For graph classes $\mathcal{G}$ and $\mathcal{H}$, we set $I_{\text {sos }}$ to be the ideal generated by the elements of $I_{\mathcal{G}}, I_{\mathcal{H}}$ and $I_{\mathcal{G}} \square \mathcal{H}$.

Note that our definition of $I_{\text {sos }}$ depends on the specific parameters $n_{\mathcal{G}}, n_{\mathcal{H}}, k_{\mathcal{G}}$ and $k_{\mathcal{H}}$.

THEOREM 3.5. The points in the variety $\mathcal{V}\left(I_{\text {sos }}\right)$ are in bijection to the triple of graphs whose components are in $\mathcal{G}$, in $\mathcal{H}$ and in their corresponding product graph with a dominating set of any size.

Proof. We have already demonstrated that $\mathcal{V}\left(I_{\mathcal{G}}\right), \mathcal{V}\left(I_{\mathcal{H}}\right)$ are in bijection to the graphs in $n_{\mathcal{G}}, n_{\mathcal{H}}$ vertices with minimum dominating sets of size $k_{\mathcal{G}}, k_{\mathcal{H}}$ respectively. It remains to investigate the restrictions placed on the $x_{g h}$ variables, which denote whether or not the vertex $g h \in V(\mathcal{G} \square \mathcal{H})$ appears in the dominating set of the product graph. Eqns. (2a) force the vertex variables $x_{g h}$ to be "on" or "off", i.e., the vertex is in the dominating set if $x_{g h}=1$ and is outside the dominating set otherwise. Eqns. (2b) force every vertex $g h$ to be dominated. It is either in the set itself (i.e., $1-x_{g h}=0$ ), or it is adjacent to a vertex in the dominating set via an edge from the underlying graph in $\mathcal{G}$ or the underlying graph in $\mathcal{H}$. In particular, the edge $e_{g g^{\prime}}$ is "on" and the vertex $x_{g^{\prime}} h$ is in the dominating set, or the $e_{h h^{\prime}}$ is "on" and the vertex $x_{g h^{\prime}}$ is in the dominating set. In either of these cases, the vertex $x_{g h}$ is dominated. Therefore, the points in the variety $\mathcal{V}\left(I_{\text {sos }}\right)$ are in bijection to the graphs in $n_{\mathcal{G}}, n_{\mathcal{H}}$ vertices with minimum dominating sets of size $k_{\mathcal{G}}, k_{\mathcal{H}}$ respectively, and their corresponding product graph with a dominating set of any size. 
Observe that there are no polynomials in $I_{\text {sos }}$ enforcing minimality on the dominating set in the product graph. This is essential when we tie all of these ideals and definitions together, and model Vizing's conjecture as a sum-of-squares problem. In particular, we model Vizing's conjecture as an ideal/polynomial pair, where the polynomial must be nonnegative on the variety associated with the ideal if and only if Vizing's conjecture is true.

Definition 3.6. Given the graph classes $\mathcal{G}$ and $\mathcal{H}$, define

$$
f^{*}=\left(\sum_{g h \in V(\mathcal{G}) \times V(\mathcal{H})} x_{g h}\right)-k_{\mathcal{G}} k_{\mathcal{H}} .
$$

THEOREM 3.7. Vizing's conjecture is true if and only if for all values of $n_{\mathcal{G}}, k_{\mathcal{G}}, n_{\mathcal{H}}$ and $k_{\mathcal{H}}, f^{*}$ is nonnegative on $\mathcal{V}\left(I_{\text {sos }}\right)$, i.e.,

$$
\forall z \in \mathcal{V}\left(I_{\text {sos }}\right): f^{*}(z) \geq 0 .
$$

Proof. Assume that Vizing's conjecture is true. Therefore, for all graphs $G \in \mathcal{G}$ and $H \in \mathcal{H}$, we have $\gamma(G \square H) \geq \gamma(G) \gamma(H)$. In particular, $\gamma(G \square H)-k_{\mathcal{G}} k_{\mathcal{H}} \geq 0$, for all values of $n_{\mathcal{G}}, k_{\mathcal{G}}, n_{\mathcal{H}}$ and $k_{\mathcal{H}}$. Since $f^{*}$ contains a sum over all the $x_{g h}$ variables, which represent a dominating set in $G \square H$ of any size, we have $f^{*}(z) \geq 0$ for all $z \in \mathcal{V}\left(I_{\text {sos }}\right)$.

Similarly, if $f^{*}(z) \geq 0$ for all $z \in \mathcal{V}\left(I_{\text {sos }}\right)$, every dominating set in $G \square H$ has size at least $k_{\mathcal{G}} k_{\mathcal{H}}$. In particular, the minimum dominating set in $G \square H$ has size at least $k_{\mathcal{G}} k_{\mathcal{H}}$ and Vizing's conjecture is true.

Corollary 3.8. Vizing's conjecture is true if and only if for each $n_{\mathcal{G}}, k_{\mathcal{G}}, n_{\mathcal{H}}$ and $k_{\mathcal{H}}$, there exists an integer $\ell$ such that $f^{*}$ is $\ell$-sos modulo $I_{\text {sos. }}$.

Proof. The ideal $I_{\text {sos }}$ contains the univariate polynomial $x^{2}-x$ for each variable. Therefore, by Lemma $2.3, I_{\text {sos }}$ is radical. By similar reasoning, $\mathcal{V}\left(I_{\text {sos }}\right)$ is finite. Therefore, by Lemma 2.5 , we know that if a polynomial is nonnegative on $\mathcal{V}\left(I_{\text {sos }}\right)$, there exists an integer $\ell$ such that the polynomial is $\ell$-sos modulo $I_{\text {sos. }}$.

In this section, we have drawn a parallel between Vizing's conjecture and a sum-of-squares problem. We defined the ideal/polynomial pair $\left(I_{\text {sos }}, f^{*}\right)$ such that $f^{*}(z) \geq 0$ for all $z \in \mathcal{V}\left(I_{\text {sos }}\right)$ if and only if Vizing's conjecture is true. In the next section, we describe exactly how to find these Positivstellensatz certificates of nonnegativity, or equivalently, these Positivstellensatz certificates that Vizing's conjecture is true.

\section{METHODOLOGY}

\subsection{Overview of the Methodology}

In our approach to Vizing's conjecture we "partition" the graphs $G, H$ and $G \square H$ by their sizes (number of vertices) $n_{\mathcal{G}}$ and $n_{\mathcal{H}}$ and by the sizes of their dominating sets $k_{\mathcal{G}}$ and $k_{\mathcal{H}}$. Note that we aim for certificates for all partitions as this would prove the conjecture. However in the following we present our method which works for a fixed partition (i.e. for fixed values of $n_{\mathcal{G}}, k_{\mathcal{G}}, n_{\mathcal{H}}$ and $k_{\mathcal{H}}$ ), and only later relax this and generalize to parametrized partitions.

The outline is as follows:

- Step 1: Model the graph classes as ideals

- Step 2: Formulate Vizing's conjecture as sum-of-squares existence question
- Step 3: Transform to a semidefinite program

- Step 4: Obtain a numeric certificate

- Step 5: Guess an exact certificate

- Step 6: Computationally verify the certificate

- Step 7: Generalize the certificate

- Step 8: Prove correctness

For fixed values of $n_{\mathcal{G}}, k_{\mathcal{G}}, n_{\mathcal{H}}$ and $k_{\mathcal{H}}$ the first step is to create the ideal $I_{\text {sos }}$ as described in Section 3, in particular Definition 3.4. To summarize, we create the ideal $I_{\text {sos }}$ in a suitable polynomial ring in such a way that the points in the variety $\mathcal{V}\left(I_{\text {sos }}\right)$ are in bijection to the triple of graphs whose components are $\mathcal{G}, \mathcal{H}$ and their corresponding product graph with a dominating set of any size. In this polynomial ring there is a variable for each possible edge of $\mathcal{G}$ and $\mathcal{H}$ (indicating whether this edge is present or not in a particular graph) and for each vertex of $\mathcal{G} \square \mathcal{H}$ (indicating whether it is in the dominating set or not).

The second step is to using the polynomial ring variables mentioned above to reformulate Vizing's conjecture: It is true for a fixed partition if a certain polynomial is nonnegative if evaluated at all points in the variety $\mathcal{V}\left(I_{\text {sos }}\right)$ of the constructed ideal. For showing that the polynomial is nonnegative, we aim for rewriting it as a finite sum of squares of polynomials (modulo the ideal $I_{\text {sos }}$ ). If we find such polynomials, then these form a certificate for Vizing's conjecture for the fixed partition. To be more precise and as already described in Section 3, Vizing's conjecture is true for this fixed values of $n_{\mathcal{G}}, k_{\mathcal{G}}, n_{\mathcal{H}}$ and $k_{\mathcal{H}}$ if and only if $f^{*}$ is $\ell$-sos modulo $I_{\text {sos }}$.

In the subsequent Section 4.2 we describe how to perform step three and to do another reformulation, namely as a semidefinite program. Note that in order of doing so, we need to have specified $\ell$. Note also that in order to prepare the semidefinite program, we need basis polynomials (i.e., special generators) of the ideals. These are obtained by computing a Gröbner basis of the ideal; see [8].

The fourth step (Section 4.3) is now to solve the semidefinite program. If the program is infeasible (i.e., there exists no feasible solution), we increase $\ell$. On the other hand, if the program is feasible, then we can construct a numeric sum-of-squares certificate. As the underlying system of equations-therefore the future certificate-is quite large, we iterate the following tasks: Find a numeric solution to the semidefinite program, find or guess some structure in the solution, use these new relations to reduce the size of the semidefinite program, and begin again with solving. This reduces the solution space and therefore potentially also the size (number $d$ of summands) of the certificate and the number of monomials of the $s_{i}$ from Definition 2.4. The procedure above goes hand-in-hand with our next step (Section 4.4), namely obtaining (one might call it guessing) an exact certificate out of the numeric certificate.

Once we have a candidate for an exact certificate, we can check its validity computationally by summing up the squares and reducing modulo the ideal; see our step six described in Section 4.5.

We want to point out, that we still consider Vizing's conjecture for a particular partition of graphs. However, having such certificates for some partitions, one can go for generalizing them by introducing parametrized partitions of graphs. Our seventh step in Section 4.6 provides more information.

The final step is to prove that the newly obtained, generalized certificate candidate is indeed a certificate; see Section 4.6. 


\subsection{Transform to a Semidefinite Program}

Semidefinite programming refers to the class of optimization problems where a linear function with a symmetric matrix variable is optimized subject to linear constraints and the constraint that the matrix variable must be positive semidefinite. A semidefinite program (SDP) can be solved in polynomial time. In practice the most prominent methods for solving an SDP efficiently are interior-point algorithms. We use the solver Mosek [21] within Matlab. For more details on solving SDPs and on interior-point algorithms see [26].

It is possible to check whether a polynomial $f$ is $\ell$-sos modulo an ideal with semidefinite programming. We refer to [5, pg. 298] for detailed information and examples. We will now present how to do so for our setting only.

Let us first fix (for example, by computing) a reduced Gröbner basis $B$ of $I_{\text {sos }}$ and fix a nonnegative integer $\ell$. Denote by $v$ the vector of all monomials in our polynomial ring $P$ of degree at most $\ell$ which can not be reduced ${ }^{2}$ modulo $I_{\text {sos }}$ by the Gröbner basis $B$. Let $p$ be the length of the vector $v$. Then $f^{*}$ (of Definition 3.6) is $\ell$-sos modulo $I_{\text {sos }}$ if and only if there is a positive semidefinite matrix $X \in \mathbb{R}^{p \times p}$ such that $f^{*}$ is equal to

$$
v^{T} X v
$$

when reduced over $B$. Hence the SDP we end up with optimizes the matrix variable $X \in \mathbb{R}^{p \times p}$ subject to linear constraints that guarantee the above equality. The objective function can be chosen arbitrarily because any matrix satisfying the constraints is sufficient for our purpose. More will be said on this later.

If the SDP is feasible, due to the positive semidefiniteness we can decompose the solution $X$ into $X=S^{T} S$. Then we define the polynomial $s_{i}$ by the $i$-th row of $S z$ and obtain

$$
v^{T} X v=(S v)^{T}(S v)=\sum_{i} s_{i}^{2} \equiv f^{*} \bmod I_{\text {sos }} .
$$

Note that the last congruence holds due to the constraints in the SDP. Equation (3) then certifies that $f^{*}$ can be written as a sum of squares of the $s_{i}$, and hence, $f^{*}$ is $\ell$-sos modulo $I_{\text {sos }}$ according to Definition 2.4.

If the SDP is infeasible, we know only that there is no certificate of degree $\ell$. We increase $\ell$ to $\ell+1$, because $f^{*}$ could still be $(\ell+1)$-sos modulo $I_{\text {sos }}$ or posses a certificate of even higher degree. However, if no new reduced monomials appear in this increment, then by Lemma 2.5 and Theorem 3.7 Vizing's conjecture does not hold.

Example 4.1. We consider the graph classes $\mathcal{G}$ and $\mathcal{H}$ with $n_{\mathcal{G}}=3$, $k_{\mathcal{G}}=2, n_{\mathcal{H}}=3$ and $k_{\mathcal{H}}=2$. Using SageMath [24] we construct the ideal $I_{\text {sos }}$, generated by 32 polynomials in 15 variables. Again using SageMath, we find a Gröbner basis of size 95.

First, we check the existence of a 1-sos certificate. The vector $v$ for $\ell=1$ has length 12 , i.e., we set up an SDP with a matrix variable $X \in$ $\mathbb{R}^{12 \times 12}$. Imposing the necessary constraints to guarantee $\sum_{i} s_{i}^{2} \equiv$ $f^{*} \bmod I_{\text {sos }}$ leads to 67 linear equality constraints. Interior-point algorithms detect infeasibility of this SDP in less than half a second, this implies that there is no 1-sos certificate.

Setting up the SDP for checking the existence of a 2-sos certificate results in a problem with a matrix variable of dimension 67 and 359

\footnotetext{
${ }^{2}$ Algorithmically speaking, we say that a polynomial $f$ is reduced modulo the ideal $I$ if $f$ is the representative of $f+I$ which is invariant under reduction by a reduced Gröbner basis of the ideal $I$.
}

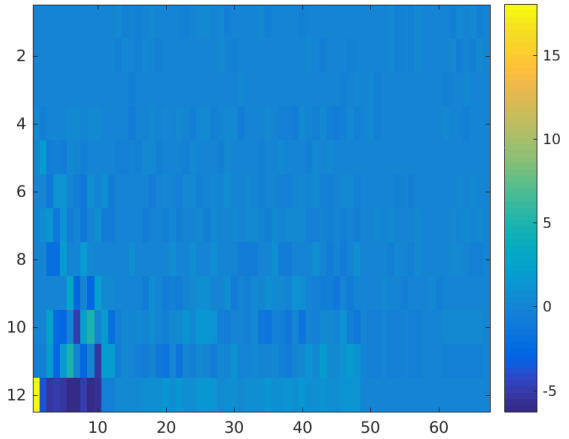

Figure 1: Plotting the entries of matrix $S . S_{i, j}$ is the coefficient of the $j$-th monomial in the $i$-th polynomial $s_{i}$ of the numeric sum-of-squares certificate.

linear constraints. Interior-point algorithms find a solution $X$ of this SDP in 0.72 seconds, this guarantees the existence of a numeric 2-sos certificate for these graph classes.

\subsection{Obtain a Numeric Certificate}

As described in Section 4.2 above, after solving the SDP we decompose the solution $X$. We do so be computing the eigenvalue decomposition $X=V^{T} D V$ and then setting $S=D^{1 / 2} V$. ( $D$ is the diagonal matrix having the eigenvalues on the main diagonal. Since $X$ is positive semidefinite, all eigenvalues are nonnegative and we can compute $D^{1 / 2}$.) The matrices $X, V$, and $D$ are obtained through numeric computations, hence there might be entries in $D$ that are rather close to zero but not considered as zero. We may try setting these almost-zero eigenvalues to zero, which reduces the number of polynomials of the sum-of-squares certificate.

Furthermore, a zero-column in $S$ means that the corresponding monomial is not needed in the certificate. Hence, we may try to compute a certificate where we remove all monomials corresponding to almost-zero columns. This can decrease the size of the SDP considerably and a smaller size of the matrix and fewer constraints is favorable for solving the SDP. Of course, if removing these monomials leads to infeasibility of the SDP, then removing these monomials was not correct.

As already mentioned we can choose the objective function arbitrarily. Our experiments show that different objective functions lead to (significantly) different solutions. Therefore, we carefully choose a suitable objective function leading to a "nice" solution for each instance.

Example 4.2. We look again at the case we considered in Example 4.1, that is $\mathcal{G}$ and $\mathcal{H}$ with $n_{\mathcal{G}}=3, k_{\mathcal{G}}=2, n_{\mathcal{H}}=3$ and $k_{\mathcal{H}}=2$, for which we already obtained an optimal solution $X$ and a numeric 2-sos certificate.

After computing (numerically) the eigenvalue decomposition $X=V^{T} D V$, we set all almost-zero eigenvalues to zero and compute $S=D^{1 / 2} V$, which results in a $12 \times 67$ matrix, i.e., 55 eigenvalues are considered as zero. In Figure 1 a heat map of matrix $S$ is displayed. It seems unattainable to convert this obtained solution to an exact certificate (see Section 4.4), so we take a different path. 


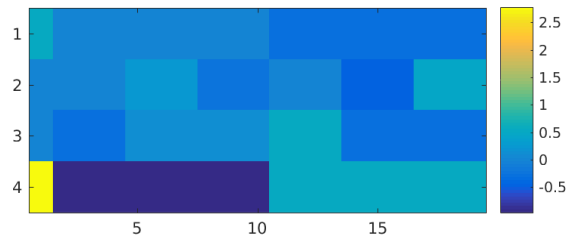

Figure 2: Plotting the entries of matrix $S$ as in Figure 1. The numeric sos certificate consists of 4 (number of rows) polynomials $s_{1}, \ldots, s_{4}$ in 19 (number of columns) monomials.

Using different objective functions and aiming for a certificate where only certain monomials appear can lead to results with a clearer structure. If the $i$-th monomial should not be included we can set the corresponding $i$-th row and column of $X$ equal to zero and obtain another SDP, where we have fewer variables and modified constraints. We now try to use only the 19 monomials $1, x_{g h}$ and $x_{g h} x_{g h^{\prime}}$ for all $g \in V(\mathcal{G})$ and all $h, h^{\prime} \neq h \in V(\mathcal{H})$.

This results in an SDP with a matrix variable of dimension 19 and 99 constraints. The SDP can be solved in 0.48 seconds, and again, we obtain matrix $S$ (after setting almost-zero eigenvalues to zero), which now is of dimension $4 \times 19$. A heat map is given in Figure 2.

As one sees in Figure 2, $S$ has a certain block structure, suggesting that in each $s_{i}$ the coefficients of the monomials depend only on the index $g \in V(\mathcal{G})$ and there is no dependence on the indices $h \in V(\mathcal{H})$. Therefore, we aim for a 2-sos certificate of the form

$$
\begin{aligned}
s_{i}=v_{i} & +\sum_{g \in V(\mathcal{G})} \lambda_{g, i}\left(\sum_{h \in V(\mathcal{H})} x_{g h}\right) \\
& +\sum_{g \in V(\mathcal{G})} \mu_{g, i}\left(\sum_{\left\{h, h^{\prime}\right\} \subseteq V(\mathcal{H})} x_{g h} x_{g h^{\prime}}\right)
\end{aligned}
$$

for $i \in\left\{1, \ldots, n_{\mathcal{G}}\right\}$ and

$$
\begin{aligned}
s_{0}=\alpha & +\beta\left(\sum_{g \in V(\mathcal{G})} \sum_{h \in V(\mathcal{H})} x_{g h}\right) \\
& +\gamma \sum_{g \in V(\mathcal{G})}\left(\sum_{\left\{h, h^{\prime}\right\} \subseteq V(\mathcal{H})} x_{g h^{x}} x_{g h^{\prime}}\right),
\end{aligned}
$$

where the coefficients $\alpha, \beta, \gamma, v_{i}, \lambda_{g, i}$ and $\mu_{g, i}$ are the entries of $S$. However, we only have the numeric values

$$
S=\left(\begin{array}{rrrrrrr}
0.535 & 0.011 & 0.011 & 0.011 & -0.289 & -0.289 & -0.289 \\
0.000 & 0.000 & 0.236 & -0.236 & -0.001 & -0.471 & 0.472 \\
-0.000 & -0.272 & 0.136 & 0.136 & 0.544 & -0.273 & -0.272 \\
2.778 & -0.962 & -0.962 & -0.962 & 0.536 & 0.536 & 0.536
\end{array}\right)
$$

at hand and it is not obvious how to guess suitable exact numbers from it. In contrast, looking at the values

$$
X=\left(\begin{array}{rrrrrrr}
8.000 & -2.667 & -2.667 & -2.667 & 1.333 & 1.333 & 1.333 \\
-2.667 & 1.000 & 0.889 & 0.889 & -0.667 & -0.444 & -0.444 \\
-2.667 & 0.889 & 1.000 & 0.889 & -0.444 & -0.667 & -0.444 \\
-2.667 & 0.889 & 0.889 & 1.000 & -0.444 & -0.444 & -0.667 \\
1.333 & -0.667 & -0.444 & -0.445 & 0.667 & 0.222 & 0.222 \\
1.333 & -0.444 & -0.667 & -0.445 & 0.222 & 0.667 & 0.222 \\
1.333 & -0.444 & -0.444 & -0.667 & 0.222 & 0.222 & 0.667
\end{array}\right) \text {, }
$$

it seems almost obvious which simple algebraic numbers the entries of $X$ could be, e.g. $0.667=2 / 3$. We will use that in the following section.

\subsection{Guess an Exact Certificate}

We now have a guess for the structure of the certificate, but coefficients that are simple algebraic numbers are hard to determine from the numbers in $S$. On the other hand, the exact numbers in $X$ seem to be rather obvious so we go back to the relation $X=S^{T} S$. It implies that if we fix two monomials then the inner product of the vectors of the coefficients of these monomials in all the $s_{i}$ has to be equal to the corresponding number in $X$.

Setting up a system of equations using all possible inner products, we may obtain a solution to this system. This solution determines the coefficients in the certificate (and the certificate might be simplified even further).

Example 4.3. We continue Example 4.1, that is we consider the graph classes $\mathcal{G}$ and $\mathcal{H}$ with $n_{\mathcal{G}}=3, k_{\mathcal{G}}=2, n_{\mathcal{H}}=3$ and $k_{\mathcal{H}}=2$.

The exact numbers in $X$ given in Example 4.2 can be guessed easily. In fact, if this guess for $X$ is correct, every choice of $S$ such that $S^{T} S=X$ gives a certificate. Using the relation $S^{T} S=X$ we set up a system of equations on the parameters of (4). To be more

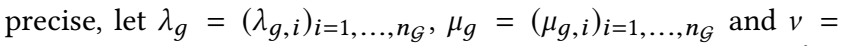
$\left(v_{i}\right)_{i=1, \ldots, n_{\mathcal{G}}}$. Then we can define the vectors $a=\left(\begin{array}{c}v \\ \alpha\end{array}\right), b_{g}=\left(\begin{array}{c}\lambda_{g} \\ \beta\end{array}\right)$ and $c_{g}=\left(\begin{array}{c}\mu_{g} \\ \gamma\end{array}\right)$, and $S^{T} S=X$ (together with the guessed values for $X)$ implies that

$$
\begin{aligned}
\langle a, a\rangle & =2\left(n_{\mathcal{G}}-1\right)^{2}, & r l & \\
\left\langle a, b_{g}\right\rangle & =-\frac{4}{3}\left(n_{\mathcal{G}}-1\right), & \left\langle a, c_{g}\right\rangle & =\frac{2}{3}\left(n_{\mathcal{G}}-1\right), \\
\left\langle b_{g}, b_{g}\right\rangle & =1, & \left\langle b_{g}, b_{g^{\prime}}\right\rangle & =\frac{8}{3}, \\
\left\langle c_{g}, c_{g}\right\rangle & =\frac{6}{9}, & \left\langle c_{g}, c_{g^{\prime}}\right\rangle & =\frac{2}{9}, \\
\left\langle b_{g}, c_{g}\right\rangle & =-\frac{6}{9}, & \left\langle b_{g}, c_{g^{\prime}}\right\rangle & =-\frac{4}{9}
\end{aligned}
$$

has to hold for each $g \in V(\mathcal{G})$, where $\langle\cdot, \cdot\rangle$ denotes the standard inner product. Under the assumption that our guess for $X$ was correct, each solution to this system of equations leads to a valid sum-of-squares certificate ( $4 \mathrm{a}$ and $4 \mathrm{~b}$ ).

We want a sparse certificate and the numeric solution suggests that $v_{2}=v_{3}=0$ holds, so we try to obtain a solution with also $v_{1}=0$ (even though the numeric solution does not fit into that setting). Using these values, the equations involving the vector $a$ determine the exact values for $\alpha, \beta$ and $\gamma$ as $\alpha=\sqrt{2}\left(n_{\mathcal{G}}-1\right), \beta=-\frac{2}{3} \sqrt{2}$ and $\gamma=\frac{1}{3} \sqrt{2}$. With that, the system of equations simplifies to

$$
\begin{array}{llrl}
\left\langle\lambda_{g}, \lambda_{g}\right\rangle & =\frac{1}{9}, & \left\langle\lambda_{g}, \lambda_{g^{\prime}}\right\rangle=0, \\
\left\langle\mu_{g}, \mu_{g}\right\rangle=\frac{4}{9}, & \left\langle\mu_{g}, \mu_{g^{\prime}}\right\rangle=0, \\
\left\langle\lambda_{g}, \mu_{g}\right\rangle=-\frac{2}{9}, & \left\langle\lambda_{g}, \mu_{g^{\prime}}\right\rangle=0 .
\end{array}
$$

Calculating $\sum_{i=1}^{n_{\mathcal{G}}}\left(s_{i}\right)^{2}$ we find out that, due to the system of equations, the sum-of-squares simplifies to

$$
\sum_{i=1}^{n_{\mathcal{G}}}\left(s_{i}\right)^{2}=\frac{1}{9} \sum_{g \in V(\mathcal{G})}\left(\left(\sum_{h \in V(\mathcal{H})} x_{g h}\right)-2\left(\sum_{\left\{h, h^{\prime}\right\} \subseteq V(\mathcal{H})} x_{g h} x_{g h^{\prime}}\right)\right)^{2} .
$$


Hence, if (4) is a sum-of-squares certificate then also

$$
\begin{aligned}
s_{0}=\alpha & +\beta\left(\sum_{g \in V(\mathcal{G})} \sum_{h \in V(\mathcal{H})} x_{g h}\right) \\
& +\gamma\left(\sum_{g \in V(\mathcal{G})} \sum_{\left\{h, h^{\prime}\right\} \subseteq V(\mathcal{H})} x_{g h^{x}} x_{g h^{\prime}}\right), \\
s_{g}= & \frac{1}{3}\left(\sum_{h \in V(\mathcal{H})} x_{g h}-2 \sum_{\left\{h, h^{\prime}\right\} \subseteq V(\mathcal{H})} x_{g h^{x}} x_{g h^{\prime}}\right) \text { for } g \in V(\mathcal{G}),
\end{aligned}
$$

where $\alpha=\sqrt{2}\left(n_{\mathcal{G}}-1\right), \beta=-\frac{2}{3} \sqrt{2}$ and $\gamma=\frac{1}{3} \sqrt{2}$ is a sum-of-squares certificate.

\subsection{Computationally Verify the Certificate}

When a certificate is conjectured, it is straightforward to verify it computationally via SageMath. To do so, it is necessary to compute the Gröbner basis of $I_{\text {sos }}$. Observe that at this point, semidefinite programming is no longer needed.

Example 4.4. We computationally verified (using SageMath) the certificate derived in Example 4.3 for the graph classes $\mathcal{G}$ and $\mathcal{H}$ with $n_{\mathcal{G}}=3, k_{\mathcal{G}}=2, n_{\mathcal{H}}=3$ and $k_{\mathcal{H}}=2$.

\subsection{Generalize the Certificate and Prove Correctness}

In Sections 4.2 to 4.5 , we presented a methodology for obtaining a sum-of-squares certificate for graph classes $\mathcal{G}$ and $\mathcal{H}$ with fixed parameters $n_{\mathcal{G}}, k_{\mathcal{G}}, n_{\mathcal{H}}$ and $k_{\mathcal{H}}$. Assuming that the previously found pattern generalizes, one can iterate the steps outlined above to obtain certificates for larger classes of graphs.

Example 4.5. We want to generalize the certificate for the graph classes $\mathcal{G}$ and $\mathcal{H}$ with $n_{\mathcal{G}}=3, k_{\mathcal{G}}=2, n_{\mathcal{H}}=3$ and $k_{\mathcal{H}}=2$ to the case $k_{\mathcal{G}}=n_{\mathcal{G}}-1, n_{\mathcal{H}}=3$ and $k_{\mathcal{H}}=2$ for $n_{\mathcal{G}} \geq 3$.

Solving the SDP for the cases $n_{\mathcal{G}}=4$ and $n_{\mathcal{G}}=5$ again yields nicely structured matrices and in fact, all the calculations done for the case $n_{\mathcal{G}}=3$ (which we already wrote down parametrized by $n_{\mathcal{G}}$ above) go through. Hence, we are able to generalize the sum-of-squares certificate (5) in the following way.

Theorem 4.6. For $k_{\mathcal{G}}=n_{\mathcal{G}}-1, n_{\mathcal{H}}=3$ and $k_{\mathcal{H}}=2$ Vizing's conjecture is true as the polynomials

$$
\begin{aligned}
s_{0}=\alpha & +\beta\left(\sum_{g \in V(\mathcal{G})} \sum_{h \in V(\mathcal{H})} x_{g h}\right) \\
& +\gamma\left(\sum_{g \in V(\mathcal{G})} \sum_{\left\{h, h^{\prime}\right\} \subseteq V(\mathcal{H})} x_{g h^{x}} x_{g h^{\prime}}\right)
\end{aligned}
$$

and

$$
s_{g}=\frac{1}{3}\left(\sum_{h \in V(\mathcal{H})} x_{g h}-2 \sum_{\left\{h, h^{\prime}\right\} \subseteq V(\mathcal{H})} x_{g h} x_{g h^{\prime}}\right) \quad \text { for } g \in V(\mathcal{G}),
$$

where $\alpha=\sqrt{2}\left(n_{\mathcal{G}}-1\right), \beta=-\frac{2}{3} \sqrt{2}$ and $\gamma=\frac{1}{3} \sqrt{2}$, are a sum-of-squares certificate with degree 2 of $f^{*}$.

The proof is not included here for space considerations. Of course, once having the theorem above, it can be verified computationally for particular parameter values, e.g. for $k_{\mathcal{G}}=4$ and $n_{\mathcal{G}}=5$, where the computation of a Gröbner basis is feasible.

\section{FURTHER EXACT CERTIFICATES}

In the previous section we saw by an example how to use our machinery combined with clever guessing in order to obtain sumof-squares certificates for proving that Vizing's conjecture holds for fixed values of $n_{\mathcal{G}}, k_{\mathcal{G}}, n_{\mathcal{H}}$ and $k_{\mathcal{H}}$, and how this can be used to obtain certificates for a less restricted set of parameters. We will use this section now in order to present further certificates that we found using the method above and for which we were able to prove correctness. Again we omit proofs due to space limitations.

\subsection{Certificates for $k_{\mathcal{G}}=n_{\mathcal{G}}$ and $k_{\mathcal{H}}=n_{\mathcal{H}}-1$}

The easiest case is the one with $k_{\mathcal{G}}=n_{\mathcal{G}}$ and $k_{\mathcal{H}}=n_{\mathcal{H}}-1$. We found the following sum-of-squares certificate and therefore know, that Vizing's conjecture holds in this case.

Theorem 5.1. For $k_{\mathcal{G}}=n_{\mathcal{G}} \geq 2$ and $k_{\mathcal{H}}=n_{\mathcal{H}}-1 \geq 2$, Vizing's conjecture is true as the polynomials

$$
s_{g}=\left(\sum_{h \in V(\mathcal{H})} x_{g h}\right)-k_{\mathcal{H}} \quad \text { for } g \in V(\mathcal{G}),
$$

are a 1-sos certificate of $f^{*}$.

Note that the certificate of Theorem 5.1 has the lowest degree possible and furthermore only uses very particular monomials of degree at most 1.

\subsection{Certificates for $k_{\mathcal{G}}=n_{\mathcal{G}}$ and $k_{\mathcal{H}}=n_{\mathcal{H}}-2$}

The next slightly more difficult case is the one for $k_{\mathcal{G}}=n_{\mathcal{G}}$ and $k_{\mathcal{H}}=n_{\mathcal{H}}-2$. Also in this case we were able to find a certificate.

THEOREM 5.2. For $k_{\mathcal{G}}=n_{\mathcal{G}} \geq 2$ and $k_{\mathcal{H}}=n_{\mathcal{H}}-2 \geq 2$, Vizing's conjecture is true as the polynomials

$s_{g}=\alpha+\beta\left(\sum_{h \in V(\mathcal{H})} x_{g h}\right)+\gamma\left(\sum_{\left\{h, h^{\prime}\right\} \subseteq V(\mathcal{H})} x_{g h} x_{g h^{\prime}}\right) \quad$ for $g \in V(\mathcal{G})$, with

$$
\begin{aligned}
& \alpha=\left(n_{\mathcal{H}}-2\right)\left(n_{\mathcal{H}}+\frac{1}{2}\left(n_{\mathcal{H}}-1\right) \sqrt{2}\right) \\
& \beta=-\left(\left(2 n_{\mathcal{H}}-3\right)+\left(n_{\mathcal{H}}-2\right) \sqrt{2}\right) \\
& \gamma=2+\sqrt{2}
\end{aligned}
$$

are a 2-sos certificate of $f^{*}$.

We want to point out, that this theorem is true whenever $\alpha, \beta, \gamma$ are solutions to the system of equations

$$
\begin{aligned}
-\left(n_{\mathcal{H}}-2\right)=\alpha^{2} & +\frac{1}{4} n_{\mathcal{H}}\left(n_{\mathcal{H}}-1\right)\left(n_{\mathcal{H}}-2\right)\left(3 n_{\mathcal{H}}-5\right) \gamma^{2} \\
& +n_{\mathcal{H}}\left(n_{\mathcal{H}}-1\right)\left(n_{\mathcal{H}}-2\right) \beta \gamma \\
1=\beta^{2} & +2 \alpha \beta-\left(n_{\mathcal{H}}-1\right)\left(n_{\mathcal{H}}-2\right)\left(2 n_{\mathcal{H}}-3\right) \gamma^{2} \\
& -3\left(n_{\mathcal{H}}-1\right)\left(n_{\mathcal{H}}-2\right) \beta \gamma \\
0=2 \beta^{2} & +2 \alpha \gamma+\left(1+3\left(n_{\mathcal{H}}-1\right)\left(n_{\mathcal{H}}-2\right)\right) \gamma^{2} \\
& +2\left(3 n_{\mathcal{H}}-4\right) \beta \gamma
\end{aligned}
$$

and that in Theorem 5.2 one particular easy solution is stated. 
Note that for all computationally considered instances, the SDP for $\ell=1$ was infeasible, so for all of those instances there is no 1 -sos certificate and one really needs monomials of degree 2 in the $s_{i}$ in order to obtain a certificate. Nevertheless, degree 2 is still very low. Furthermore also in this sum-of-squares certificate only very particular monomials are used; it can be considered sparse therefore. This is confirmed by the following example.

Example 5.3. If we consider the case $k_{\mathcal{G}}=n_{\mathcal{G}}=4, n_{\mathcal{H}}=5$ and $k_{\mathcal{H}}=3$, there are 432 monomials of degree at most 2 but the certificate of Theorem 5.2 uses only 61 of them.

\subsection{Certificates for $k_{\mathcal{G}}=n_{\mathcal{G}}$ and $k_{\mathcal{H}}=n_{\mathcal{H}}-j$}

When taking a closer look at the certificates in Theorem 5.1 and Theorem 5.2, there seems to be a structure in the certificates we found so far. In particular the certificate for the case $k_{\mathcal{G}}=n_{\mathcal{G}}$ and $k_{\mathcal{H}}=n_{\mathcal{H}}-j$ seems to be a $j$-sos certificate. Hence the following conjecture intuitively seems to be the generalization.

Conjecture 5.4. Let $k_{\mathcal{G}}=n_{\mathcal{G}}$ and $k_{\mathcal{H}}=n_{\mathcal{H}}-j$ for $j \geq 3$. Then

$$
s_{g}=\sum_{q=0}^{j} \alpha_{q}\left(\sum_{\substack{S \subseteq V(\mathcal{H}) \\|S|=q}} \prod_{h \in S} x_{g h}\right) \quad \text { for } g \in V(\mathcal{G}),
$$

where $\alpha_{q}$ are the solutions to a certain system of polynomial equations, are a $j$-sos certificate of $f^{*}$.

\section{CONCLUSIONS AND FUTURE WORK}

In this project, we modeled Vizing's conjecture as an ideal/polynomial pair such that the polynomial was nonnegative on the variety of a particularly constructed ideal if and only if Vizing's conjecture was true. We were able to produce low-degree, sparse Positivstellensätze certificates of nonnegativity for certain classes of graphs using an innovative collection of techniques ranging from semidefinite programming to clever guesswork to computer algebra. For example, Vizing's conjecture with parameters $k_{\mathcal{G}}=n_{\mathcal{G}}$ and $k_{\mathcal{H}}=n_{\mathcal{H}}-1$ has a 1-sum-of-squares Positivstellensatz and with parameters $k_{\mathcal{G}}=n_{\mathcal{G}}$ and $k_{\mathcal{H}}=n_{\mathcal{H}}-2$ has a 2-sum-of-squares Positivstellensatz. We have conjectured a broader combinatorial pattern based on these certificates, but proving validity is left to future work. However, at this time, we have indeed proved Vizing's conjecture for several classes of graphs using sum-of-squares certificates. Although we have not advanced what is currently known about Vizing's conjecture, we have introduced a completely new technique (still to be thoroughly explored) to the literature of possible approaches.

For future work, we intend to continue pushing the computational aspect of this project. Additionally, it is very easy to change the model from a Positivstellensatz certificate to a Hilbert's Nullstellensatz certificate, and thus change from numeric semidefinite programming to exact arithmetic linear algebra. This approach must also be thoroughly investigated. Finally, it would be very interesting to conjecture a global relationship between the values of $n_{\mathcal{G}}, n_{\mathcal{H}}, k_{\mathcal{G}}$ and $k_{\mathcal{H}}$, and the degree of the Positivstellensatz certificate, and perhaps even recast the conjecture in terms of the theta body hierarchy described in [11].

\section{ACKNOWLEDGMENTS}

The authors gratefully acknowledge the support of Fulbright Austria (via a Visiting Professorship at AAU Klagenfurt). This project has received funding from the European Union's Horizon 2020 research and innovation programme under the Marie SkłodowskaCurie grant agreement No 764759 and the Austrian Science Fund (FWF): I 3199-N31.

\section{REFERENCES}

[1] N. Alon and M. Tarsi. 1992. Colorings and orientations of graphs. Combinatorica 12 (1992), 125-134.

[2] B. Barak, S. Hopkins, J. Kelner, P. Kothari, and A. Potechin. 2016. A Nearly Tight Sum-of-Squares Lower Bound for the Planted Clique Problem. In FOCS 2016 IEEE 57th Annual Symposium on Foundations of Computer Science.

[3] B. Barak, P. K. Kothari, and D. Steurer. 2017. Quantum entanglement, sum of squares, and the log rank conjecture. In Proceedings of the 49th annual ACM SIGACT symposium on theory of computing, STOC '17, Montreal, QC, Canada, June 19-23, 2017. New York, NY: Association for Computing Machinery (ACM), 975-988.

[4] A. Barcalkin and L. German. 1979. The external stability number of the cartesian product of graphs. Bul. Akad. Stiinte RSS Moldoven. 1, 94 (1979), 5-8.

[5] G. Blekherman, P. A. Parrilo, and R. R. Thomas (Eds.). 2013. Semidefinite optimization and convex algebraic geometry. Philadelphia, PA: Society for Industrial and Applied Mathematics (SIAM). xix + 476 pages.

[6] B. Brešar, P. Dorbec, W. Goddard, B. L. Hartnell, M. A. Henning, S. Klavžar, and D. F. Rall. 2012. Vizing's conjecture: a survey and recent results. f. Graph Theory 69, 1-2 (2012), 46-76.

[7] W. E. Clark and S. Suen. 2000. An inequality related to Vizing's conjecture. Electron. 7. Comb. 7, 1 (2000), research paper n4, 3.

[8] D. A. Cox, J. Little, and D. O'Shea. 2007. Ideals, Varieties, and Algorithms: An Introduction to Computational Algebraic Geometry and Commutative Algebra. Springer-Verlag New York, Inc., Secaucus, NJ, USA.

[9] K. G. Fischer. 1988. Symmetric polynomials and Hall's theorem. Discrete Math 69, 3 (1988), 225-234.

[10] M. Gasca and T. Souer. 2012. Polynomial Interpolation in Several Variables. Advances in Computational Mathematics 12, 4 (2012), 377-410.

[11] J. Gouveia, P. Parrilo, and R. Thomas. 2010. Theta Bodies for Polynomial Ideals. SIAM Journal of Optimization 20, 4 (2010), 2097-2118.

[12] D. Grigoriev and N. Vorobjov. 2001. Complexity of Null- and Positivstellensatz proofs. Annals of Pure and Applied Logic 113, 1-3 (2001), 153-160.

[13] B. Hartnell and D. Rall. 1998. Domination in cartesian products: Vizing's conjecture. In Domination in graphs. Mono. Textbooks Pure and Appl. Math, Dekker, New York, 163-189.

[14] C. J. Hillar and T. Windfeldt. 2008. Algebraic characterization of uniquely vertex colorable graphs. 7. Comb. Theory, Ser. B 98, 2 (2008), 400-414.

[15] Z. Jarvis-Wloszek, R. Feeley, W. Tan, K. Sun, and A. Packard. 2005. Control Applications of Sum of Squares Programming. Lecture Notes in Control and Information Science 312 (2005), 3-22.

[16] M. Kreuzer and L. Robbiano. 2000. Computational commutative algebra. I. Berlin: Springer. $\mathrm{x}+321$ pages.

[17] M. Laurent. 2008. Sums of Squares, Moment Matrices and Optimization Over Polynomials. The IMA Volumes in Mathematics and its Applications 149 (2008), 157-270.

[18] J. A. De Loera, J. Lee, S. Margulies, and S. Onn. 2009. Expressing Combinatorial Optimization Problems by Systems of Polynomial Equations and the Nullstellensatz. Combinatorics, Probability and Computing 18, 4 (2009), 551-582.

[19] L. Lovász. 1994. Stable sets and polynomials. Discrete Mathematics 124 (1994), 137-153.

[20] S. Margulies and I. V. Hicks. 2012. An algebraic exploration of dominating sets and Vizing's conjecture. Electron. f. Comb. 19, 2 (2012), research paper p1, 30.

[21] MOSEK ApS. 2017. The MOSEK optimization toolbox for MATLAB manual. Version 8.1. http://docs.mosek.com/8.1/toolbox/index.html

[22] S. Onn. 2004. Nowhere-zero flow polynomials. Journal of Combinatorial Theory, Series A 108, 2 (2004), 205-215.

[23] L. Sun. 2004. A result on Vizing's conjecture. Discrete Mathematics 275, 1 (2004), 363-366.

[24] The Sage Developers. 2017. SageMath, the Sage Mathematics Software System (Version 8.0). https: //www. sagemath.org.

[25] V. Vizing. 1968. Some unsolved problems in graph theory. Uspekhi Mat. Nauk 23 (1968), 117-134.

[26] H. Wolkowicz, R. Saigal, and L. Vandenberghe (Eds.). 2000. Handbook of semidefinite programming. Theory, algorithms, and applications. Vol. 27. Springer, Cham. 